\title{
Hyperintense Basilar Artery on FLAIR MR Imaging: Diagnostic Accuracy and Clinical Impact in Patients with Acute Brain Stem Stroke
}

\author{
M. Gawlitza, U. Quäschling, C. Hobohm, J. Otto, P. Voigt, K.-T. Hoffmann, and D. Lobsien
}

\begin{abstract}
BACKGROUND AND PURPOSE: FLAIR-hyperintense vessels are known to be a sign of sluggish collateral blood flow in hemispheric vessel occlusion. Additionally, they seem to have a prognostic implication. The aim of the current study was to evaluate the hyperintense configuration of the basilar artery (FLAIR-hyperintense basilar artery) as a marker of basilar artery occlusion and as a predictor of patient outcome.
\end{abstract}

MATERIALS AND METHODS: We retrospectively identified 20 patients with basilar artery occlusion who initially underwent MR imaging with subsequent DSA. The diagnostic accuracy of the FLAIR-hyperintense basilar artery sign was tested by 4 independent readers in a case-control design, and the relation among FLAIR-hyperintense basilar artery and DWI posterior circulation-ASPECTS, patient outcome, and patient survival was evaluated. To grade the extent of the FLAIR-hyperintense basilar artery sign, we generated a score by counting the number of sections from the basilar tip to the foramen magnum in which a hyperintense signal in the vessel lumen was present multiplied by the section thickness.

RESULTS: The FLAIR-hyperintense basilar artery sign showed moderate sensitivity (65\%-95\%) but very good specificity ( $95 \%-100 \%)$ and accuracy (85\%-93\%) for the detection of basilar artery occlusion. Substantial or excellent inter-reader agreement was observed (Cohen $\kappa$, $0.64-0.85)$. The FLAIR-hyperintense basilar artery inversely correlated with the posterior circulation-ASPECTS $(r=-0.67, P=.01)$. Higher FLAIR-hyperintense basilar artery scores were associated with patient death $(28.3 \pm 13.7$ versus $13.4 \pm 11.1, P<.05)$.

CONCLUSIONS: The FLAIR-hyperintense basilar artery sign proved to be a valuable marker of vessel occlusion and may substantially support the diagnosis of basilar artery occlusion. The established FLAIR-hyperintense basilar artery score may be helpful for the prediction of individual patient survival.

ABBREVIATIONS: BAO = basilar artery occlusion; FHBA = FLAIR-hyperintense basilar artery; FHV = FLAIR-hyperintense vessel; pc-ASPECTS = posterior circulation-ASPECTS

$\mathbf{F}^{1}$ LAIR-hyperintense vessels (FHVs) are frequently observed in the M2-to-M4 segments of patients with acute ischemic stroke of the anterior circulation. They can be an indicator of occlusion, ${ }^{1,2}$ reversible constriction, ${ }^{3}$ or stenosis ${ }^{4-6}$ of intra- and extracranial arteries, and they are identified as the absence of the typical "flow void" in the tortuous sulcal arteries on the cerebral surface. ${ }^{2,7}$ It is hypothesized that the FHV sign is caused mainly by sluggish, slow blood flow and also by clot signal intensity, the latter as an effect of oxyhemoglobin.,

Received November 20, 2013; accepted after revision January 13, 2014

From the Departments of Neuroradiology (M.G., U.Q., K.-T.H., D.L.), Diagnostic and Interventional Radiology (M.G., J.O., P.V.), and Neurology (C.H.), University Hospital Leipzig, Leipzig, Germany.

Please address correspondence to Matthias Gawlitza, MD, Department of Neuroradiology, University Hospital Leipzig, Liebigstrasse 20, 04103 Leipzig, Germany;

e-mail: matthias.gawlitza@medizin.uni-leipzig.de

三 Indicates article with supplemental on-line table.

http://dx.doi.org/10.3174/ajnr.A3961
At the beginning, the FHV sign was mainly proposed as a very sensitive marker of vessel occlusion and of flow impairment in MCA stroke. ${ }^{8,10-14}$ Publications dealing with its prognostic significance were rare $^{8,15}$ until Lee et al, ${ }^{1}$ in 2009 , observed a relation between the extent of the FHV and the amount of diffusion-perfusion mismatch in patients with MCA occlusions. They suggested that the FHV is an indicator of collateral flow besides its proved sensitivity for mere blood flow alterations. Since then, there have been several original studies $^{3,5,7,16-22}$ and 1 review $^{2}$ addressing the potential role of the FHV sign as an imaging biomarker of collateral circulation and as a predictor of patient outcome. Although some patients with basilar artery occlusion (BAO) in the low single-digit range were included in a few studies dealing with the diagnostic significance of the FHV sign, ${ }^{10,12,14}$ the focus of these investigations has been on patients with MCA stroke. To date, neither the diagnostic nor the prognostic value of the FLAIR-hyperintense basilar artery (FHBA) sign has been investigated in a dedicated study, to our knowledge. 


\section{MATERIALS AND METHODS Subjects}

In a retrospective analysis, all patients with the diagnosis of an acute basilar artery occlusion in our department from January 2003 to January 2013 were identified. We included only patients with the following: proved occlusion of the basilar artery by DSA and/or contrast-enhanced MRA; DSA, at most, 12 hours from diagnosis; and MR imaging, including axial FLAIR and DWI sequences before DSA. Information about patient survival was gathered; if available, the modified Rankin Scale scores at discharge were assessed to evaluate patient outcome. mRS 0-2 was defined as favorable, and mRS 3-6, as unfavorable outcome. Detailed patient characteristics can be found in On-line Table 1. The diagnostic performance of the FHBA was tested by defining a control group: One control patient without BAO who had undergone MR imaging for suspected stroke, matched for age, sex, and MR imaging scanner type used, was randomly selected for each patient with proved $\mathrm{BAO}$ from the PACS, to reduce the pretest probability of the FHBA and to simulate a more realistic condition with patients examined for stroke without confirmation of BAO. ${ }^{23}$ In 1 patient of the control group, an occlusion of the internal carotid artery with acute infarction in the MCA territory was present. The other patients in the control group showed no ischemia or acute infarction.

\section{MR Imaging Protocol}

Imaging was performed on either a 3T scanner (Trio; Siemens, Erlangen, Germany) or on a 1.5T MR imaging scanner (Gyroscan Intera; Philips Healthcare, Best, the Netherlands; and Magnetom Trio Symphony; Siemens) in a clinical setup with commercially available software and hardware. In most cases, a standardized stroke protocol consisting of axial DWI, axial FLAIR, axial gradient recalled-echo $\left(\mathrm{T} 2{ }^{*}\right.$ or SWI), DSC perfusion imaging, intracranial time-of-flight-angiography, and intra-/extracranial contrast-enhanced MRA was used. For DWI, echo-planar diffusion-weighted sequences with $2 \mathrm{~b}$-values $\left(0 \mathrm{~s} / \mathrm{mm}^{2}\right.$ and 1000 $\left.\mathrm{s} / \mathrm{mm}^{2}\right)$ in 3 different directions were used $(\mathrm{TR} / \mathrm{TE}=2900-6000 /$ $74-113 \mathrm{~ms}$; section thickness $=3-\mathrm{mm}$; in-plane resolution $=$ $1.4 \times 1.4-2.0 \times 2.6 \mathrm{~mm})$. FLAIR was performed with the following parameters: $\mathrm{TR} / \mathrm{TE}=8000-10,000 / 92-125 \mathrm{~ms}$; section thickness $=3-5 \mathrm{~mm}$; in-plane resolution $=0.9 \times 0.9-1.0 \times 1.5 \mathrm{~mm}$.

\section{Digital Subtraction Angiography}

DSA studies were performed, in most cases, with a biplane (Axiom Artis BA; Siemens) and, in 1 case, a monoplane angiography unit (Innova 4100 flat panel; GE Healthcare, Milwaukee, Wiscon$\sin$ ). A diagnostic angiography of at least 1 vertebral artery was performed, followed by different therapeutic endovascular interventions for recanalization of the occluded vessel (On-line Table 1).

\section{Image Analysis}

For the analysis of the FHBA sign, only the FLAIR images of patients with BAO and their randomly selected control group without BAO were independently read the by 4 readers: 2 board-certified, subspecialized neuroradiologists with 13 (U.Q., reader 1) and 8 years (D.L., reader 2) and 2 radiologists-in-training (J.O., reader 3, and P.V., reader 4) with 3 years of experience in stroke imaging, respectively. Images were reviewed on a commercially available desktop computer with Mac OS X (Apple, Cupertino, California) and an open-source freeware 4D DICOM viewer (OsiriX; http://www.osirix-viewer.com). ${ }^{24}$ Readers were fully blinded regarding patient information or MR imaging scanner. Visual hyperintensity of the basilar artery was graded on a 5-point Likert scale $(1=$ no, $2=$ little, $3=$ equivocal, $4=$ distinct, $5=$ sure). Scores of 4 and 5 were defined as positive FHBA signs. ${ }^{23}$

To grade the extent of the FHBA sign in patients with $\mathrm{BAO}$, we generated a score (called the "FHBA score" in this article) by counting the number of sections from the basilar tip down to the foramen magnum, including the dominant vertebral artery, in which a hyperintense signal in the vessel lumen was present multiplied by the section thickness (eg, 3 sections with the FHBA at a section thickness of $5 \mathrm{~mm}$ would result in a score of 15). A possible elongation of the basilar artery, resulting in a diagonal cut of the vessel by the plane of the FLAIR sequence, was not taken into account. These measurements were performed in consensus by 2 readers with 8 (D.L.) and 4 years' (M.G.) experience in neuroradiologic imaging. The same readers calculated the posterior circulation-ASPECTS (pc-ASPECTS) on DWI sequences as published by Puetz et $\mathrm{al}^{25}$ and Tei et al. ${ }^{26}$ We subdivided the site of the $\mathrm{BAO}$ into proximal, middle, or distal, taking into account all available diagnostic modalities (ie, DSA, MR imaging, and, in several cases, CT). ${ }^{27}$ We evaluated DSA datasets, grading the extent of vessel occlusion and subsequent revascularization by the TICI scale with TICI $2 \mathrm{~b}$ and TICI 3 being defined as a successful reperfusion result. ${ }^{28}$

\section{Statistical Analysis}

Statistical analysis and graphics creation were performed with the Statistical Package for the Social Sciences, Version 20 (IBM, Armonk, New York) and GraphPad Prism 5 software (GraphPad Software, San Diego, California). The $\chi^{2}$ test was used to compare categoric or nominal data, whereas continuous data were compared by using a Student unpaired $t$ test and 1-way ANOVA. Pearson $r$ was calculated for correlation analysis, in which $0-0.2$ was defined as poor; $0.2-0.4$, as weak; $0.4-0.6$, as moderate; $0.6-$ 0.8 , as strong; and $0.8-1.0$, as a very strong correlation. Interreader agreements were evaluated by using Cohen's $\kappa$; values of $<0.00$ indicated poor; $0.00-0.20$, slight; $0.21-0.40$, fair; $0.41-$ 0.60 , moderate; $0.61-0.80$, substantial; and $0.81-1.00$, excellent and/or almost perfect agreement. All continuous values are presented as mean $\pm \mathrm{SD}$. Receiver operating characteristic analysis was performed with the FHBA score as a test variable for the prediction of patient death and unfavorable outcome. For all tests, $P<.05$ was statistically significant.

\section{RESULTS}

\section{Patient Characteristics}

In total, 20 patients ( 7 men, 13 women) with proved BAO were included. The mean age was $65.5 \pm 17.1$ years (median, 69.0 years; range, $18-86$ years). Four occlusions were localized at the basilar apex $(20 \%) ; 12$, in the middle $(60 \%)$; and 4 , at the proximal segment $(20 \%)$. The mean time from MR imaging to DSA was $121.0 \pm 105.1$ minutes (median, 99.0 minutes; range, 50-541 
Cross table with interobserver agreements (Cohen $\kappa)$ among 4 readers $^{\mathbf{a}}$

\begin{tabular}{lcccc}
\hline & Reader 1 & Reader 2 & Reader 3 & Reader 4 \\
\hline Reader 1 & - & 0.72 & 0.74 & 0.70 \\
Reader 2 & 0.72 & - & 0.69 & 0.64 \\
Reader 3 & 0.74 & 0.69 & - & 0.85 \\
Reader 4 & 0.70 & 0.64 & 0.85 & - \\
\hline
\end{tabular}

Note:- indicates not applicable.

a The FHBA sign was classified using the coding "positive FHBA sign" versus "equivocal" and "negative FHBA sign."

minutes). The mean time from symptom onset to MR imaging was $264.3 \pm 263.9$ minutes (median, 182 minutes; range, 20-923 minutes; 4 patients with unknown time of symptom onset). On initial DSA, 16 patients presented with TICI 0 (80\%); 2 patients, with TICI 1 (10\%); 1 , with TICI 2a; and 1, with TICI 3 (patient 3 , in whom vessel occlusion was already recanalized after IV thrombolysis). After DSA and intra-arterial recanalization procedures, respectively, 13 patients (65\%) showed successful recanalization (6 patients with TICI 3, seven patients with TICI 2b), but in 7 patients $(35 \%)$, only incomplete ( 2 patients with TICI 2 a, two patients with TICI 1) or no reperfusion (3 patients with TICI 0 ) was achieved. Two complications related to DSA occurred (1 vessel perforation at the basilar apex, 1 complete in-stent thrombosis shortly after angiography), both fatal. Detailed patient records allowed retrospective mRS score calculation at discharge in 18 patients; 7 (38.9\%) had a favorable outcome (1 patient with mRS 0 , five patients with mRS 1 , one patient with mRS 2) and 11 (61.1\%) had an unfavorable outcome (2 patients with mRS 3, three patients with mRS 5, six patients with mRS 6). Information about patient survival was available in 19 of 20 patients; 13 patients survived and 6 died.

\section{Diagnostic Value of FHBA Sign}

Assigning visual hyperintensity ratings of 4 and 5 to a "positive FHBA sign" and 1, 2, and 3 to a "negative FHBA sign," we achieved the following sensitivity, specificity, and accuracy values for BAO: $70 \%, 100 \%$, and $85 \%$ for reader $1 ; 65 \%, 95 \%$, and $80 \%$ for reader $2 ; 85 \%, 95 \%$, and $90 \%$ for reader 3 ; and $90 \%, 95 \%$, and $93 \%$ for reader 4 . Substantial-to-excellent interreader agreement measures (by using Cohen $\kappa, 0.64-0.85$; Table) were observed.

\section{FHBA Sign, pc-ASPECTS, and Interacting Factors}

If we took into account all available imaging studies of the 20 patients with proved $\mathrm{BAO}$, the FHBA sign was unequivocally present in 17 (85\%). The mean FHBA score was $18.5 \pm 13.3$ (median, 15.0; range, 0-45). Regarding the FHBA score and the BAO occlusion site, no significant relationship was evident. We did not observe a relationship between the preinterventional TICI grade and the FHBA; likewise, the FHBA score had no effect on the extent of vessel recanalization. Eighteen patients (90\%) had DWI lesions on the initial MR imaging with a mean pc-ASPECTS of $6.2 \pm 2.9$ (median, 7.5; range, $0-10$ ). The FHBA and pcASPECTS showed a highly significant negative correlation ( $r=$ $-0.67 ; P=.01$; Figs $1 A, 2$, and 3 ). All 3 patients without definitive FHBA signs showed a pc-ASPECTS of 8 , each with a DWI lesion only in the pons.

\section{Patient Outcome and Survival}

There was a tendency toward a moderate correlation between the FHBA and mRS scores at discharge $(r=0.41 ; P=.09$; Fig $1 B)$, yet if one compared patients with favorable and unfavorable outcomes, no significant difference regarding mean FHBA scores between the groups was detected (Fig 1C). However, patient survival was associated with significantly lower FHBA values (13.4 \pm 11.1 versus $28.3 \pm 13.7 ; P<.05$; Fig $1 D)$. Whereas receiver operating characteristic analysis for patient outcome prediction revealed no statistically significant result (area under the curve, $0.71 ; P=.15$ ), an area under the curve of $0.81(P<.05)$ for the prediction of patient death was calculated. An optimal discrimination threshold for the FHBA score of $\geq 17.5$ was determined, which yielded a sensitivity of $83 \%$ and a specificity of $77 \%$. The odds ratio for patient death above the mentioned threshold value was 16.7.

pc-ASPECTS inversely correlated with $\mathrm{mRS}$ at discharge ( $r=$ $-0.45 ; P<.05)$, and patients with favorable outcomes had significantly higher pc-ASPECTS than those with unfavorable outcomes (7.86 \pm 1.87 versus $5.36 \pm 3.01 ; P<.05$; Fig $1 D)$; also patient survival was associated with higher pc-ASPECTS (7.54 \pm 1.66 versus $4.00 \pm 3.34 ; P<.01)$.

No significant difference regarding the postinterventional TICI grade was observed between patients with favorable and unfavorable functionality outcomes or between patients who died or survived. Likewise, the time from symptom onset to MR imaging and from MR imaging to DSA showed no statistical disparity between those groups. Although not related to patient outcome, the site of the BAO had a significant effect on patient survival; 4 patients $(100 \%)$ with proximal BAO died compared with 2 deaths if the occlusion was located in the midbasilar section (18\%) and no deaths with an occlusion at the basilar apex $(P<.01)$.

\section{DISCUSSION}

In our study population, the FHBA sign proved to be a reliable marker of BAO with moderate sensitivity $(65 \%-95 \%)$ and with very good specificity (95\%-100\%) and accuracy (85\%-93\%), which could also be correctly identified by readers with lower experience levels. Concerning the FHBA sign in BAO, the available literature is scarce and only a few publications provide information about this specific condition. In a series of 74 patients with acute stroke, the FHBA sign was negative in 3 of 5 cases with vertebrobasilar ischemia. ${ }^{7}$ In the same way, Maeda et $\mathrm{al}^{12}$ and Toyoda et $\mathrm{al}^{14}$ reported 3 cases of BAO, again, in patients with mainly MCA occlusions, with a negative FHBA sign, which they attributed to CSF pulsation artifacts in the prepontine cistern. ${ }^{14}$ However, all these studies focused on patients with occlusion of the MCA. In the anterior circulation, the sensitivity of FHV for major vascular abnormalities — with reported values as high as $94 \%,{ }^{7} 96.7 \%,{ }^{14}$ or $100 \%{ }^{11}$ - is known to be comparable with that of 3D time-of-flight MRA, ${ }^{10,29}$ but with a better accuracy and often shorter acquisition times. Cosnard et al, ${ }^{10}$ therefore, even recommended the omission of time-of-flight MRA from the routine MR imaging diagnostics of stroke. As in our dedicated study, 3 patients (15\%) with proved BAO did not show an unequivocally positive FHBA; therefore, we would not recommend that the diagnosis should be based solely on this sign.

When in doubt, additional MR angiography sequences are 

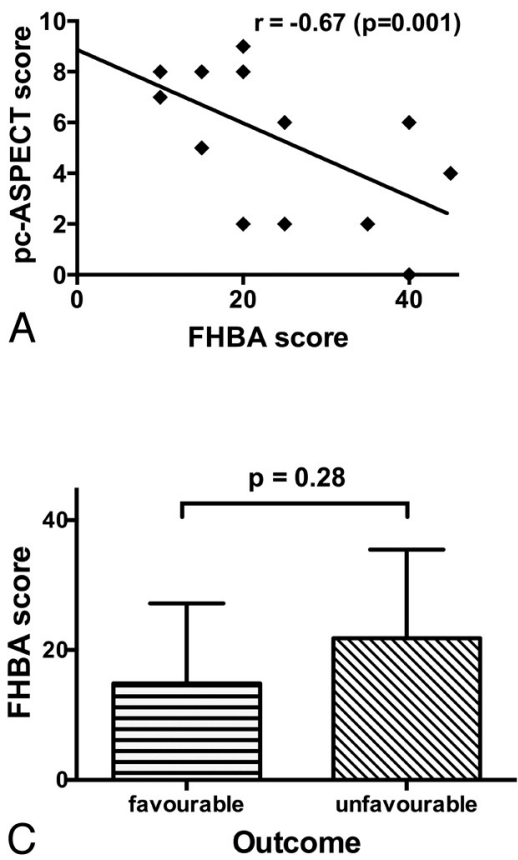

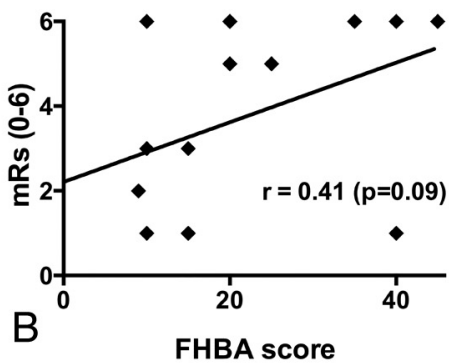

$p<0.05$

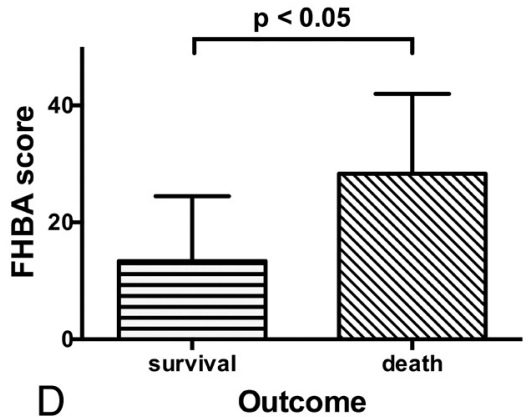

FIG 1. The FHBA score and its correlations with pc-ASPECTS $(A)$ and $\mathrm{mRS}$ scores $(B)$. $A$, A significant negative correlation between pc-ASPECTS and FHBA scores can be seen. $B$, Note a tendency for a correlation between FHBA and mRS; however, this was not significant at $P<.05$. In a comparison of FHBA between patients with favorable and unfavorable outcomes, a tendency toward higher scores with unfavorable outcomes was detected $(C)$, yet there was no significant difference. $D$, Comparison between survival and patient death. Higher FHBA scores are significantly associated with death from BAO.
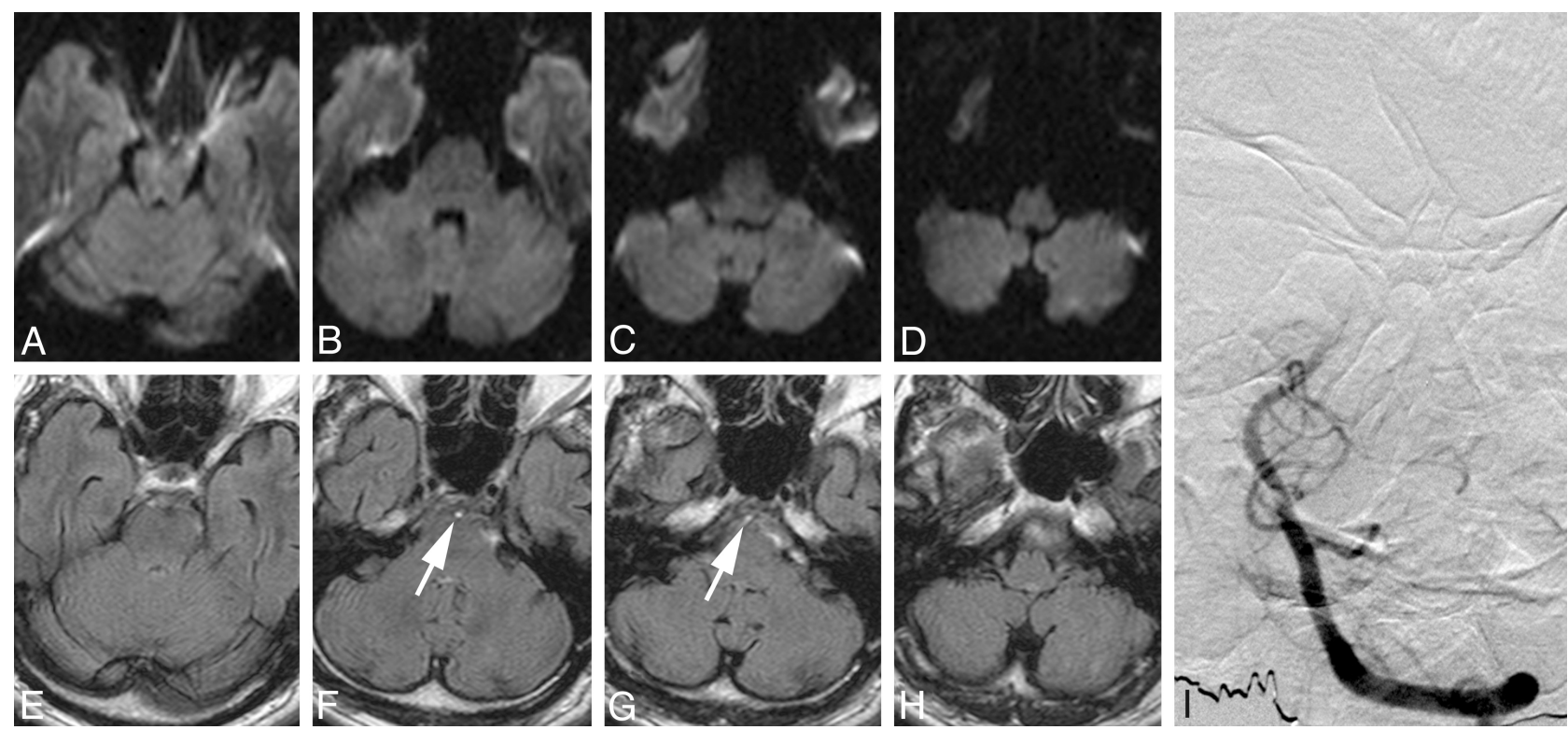

FIG 2. MR imaging and DSA of a patient with acute postural vertigo, double vision, and downbeat nystagmus. A-D, On axial DWI sections, no sign of acute infarction can be seen. $A-D$, Axial FLAIR images. $F$ and $G$, A hyperintense basilar artery can be identified (arrows) and was graded with an FHBA score of ten. I, Anteroposterior left vertebral artery angiogram. Occlusion of the middle-to-superior segment of the basilar artery is shown. After intra-arterial administration of $30-\mathrm{mg}$ rtPA, the patient recovered almost completely from his initial symptoms and left the hospital with slight residual gait ataxia (mRS 1).

necessary. ${ }^{30,31}$ Nevertheless, we think that the FHBA sign is a valuable imaging characteristic that, if present and correctly identified, may support a fast diagnosis and avoid delay of a potentially lifesaving treatment. ${ }^{32}$ The FHBA sign might be of particular importance if MR angiography is degraded by artifacts or if MR angiography is initially not scheduled, for example, in patients with atypical neurologic symptoms not suggestive of acute stroke.
Besides its diagnostic capabilities, the FHBA sign might also have prognostic relevance because high FHBA scores were associated with more extensive ischemic lesion patterns. This finding is in accordance with those in other publications that described the close relation between hyperintense vessels and larger DWI lesions in patients with stroke of the MCA territory. Also, a relation between the FHV and perfusion MR imaging was described, 

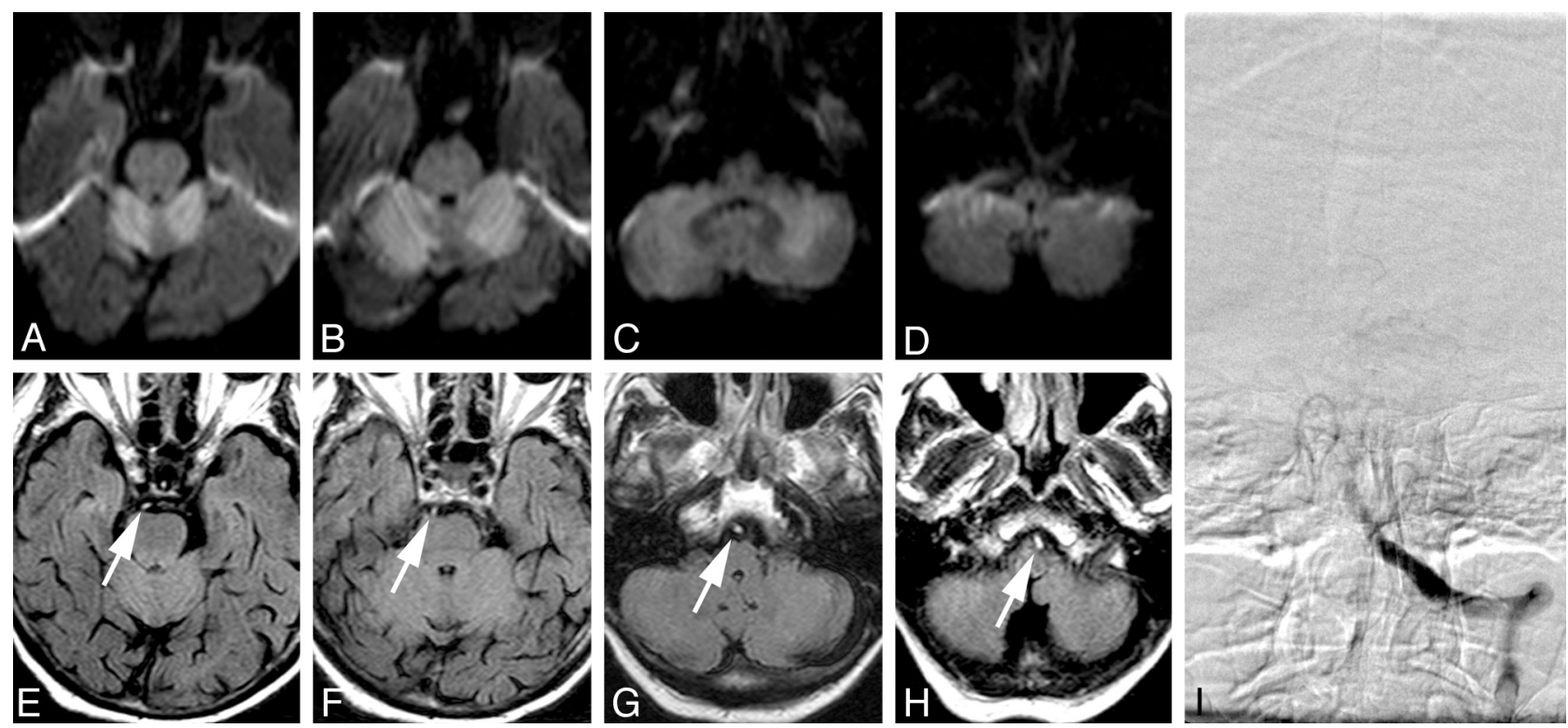

FIG 3. MR imaging and DSA of a patient who was found in a soporous state with anisocoria and fixed pupils. A-D, In axial DWI, extensive diffusion restriction (ADC maps not shown) can be identified in both cerebellar hemispheres $(A-C)$, indicating acute cerebellar infarction. $E-H$ In axial FLAIR images, a hyperintense basilar artery can be identified throughout the course of the vessel, seen as a "bright dot" anterior to the brain stem, and was graded with an FHBA score of thirty-five. I, Anteroposterior left vertebral artery angiogram. Occlusion of the proximal-tomiddle segment of the basilar artery is shown. The basilar artery could not be recanalized, and the patient died on the same day.

with a more pronounced FHV sign being associated with larger DSC perfusion imaging and mismatch volume ${ }^{33}$; FHV sign is, therefore, thought to be a marker of the amount of brain parenchyma with blood flow alteration and of potential salvageable tissue in MCA stroke. ${ }^{1,2,7,11,13}$ Analogous to this, the extent of the FHBA sign might also be a marker of impaired hemodynamics in BAO with pronounced FHBA, meaning larger brain areas being hypoperfused; this more pronounced hypoperfusion could also explain larger DWI lesions, measured by the low pc-ASPECTS in our patient group. Unfortunately, because they were not performed in all of our patients and were several times distorted by motion artifacts, we had too few reliable perfusion studies available to directly address the connection among the FHBA sign, perfusion lesion size, and mismatch volumes; and it was beyond the scope of this article to investigate the meaning of the FHBA sign as an indicator of slow flow, collateral circulation, and tissueat-risk. Furthermore, it was not the intention of the present study to relate the FHBA to the occlusion site (ie, whether it occurred proximal or distal or also at the level of the thrombus). ${ }^{34}$ By analogy with hemispheric stroke, ${ }^{7}$ the FHBA proximal to the occlusion site might be an expression of slow flow, whereas the FHBA distal to a thrombosis could be caused by retrograde perfusion of the basilar artery, for example, via the posterior communicating arteries. On the other hand, thrombus material can also be hyperintense on FLAIR images. ${ }^{35}$ Concerning this topic, research projects comparing FLAIR images with perfusion MR imaging studies and DSA are necessary. In addition, follow-up MR imaging after thrombolysis might be interesting for further studies investigating whether wall abnormalities might play a role in the etiology of FHBA.

FHBA scores between patients with poor and good functional outcomes did not differ significantly, though there was a tendency toward good functional outcomes being associated with lower
FHBA scores (Fig 1C). The small patient sample might, at least partially, explain the lack of statistical significance. However, the FHBA score was significantly higher in deceased patients than in those who survived, a result also underpinned by the receiver operating characteristic analysis. To date, the prognostic role of the FHV sign in stroke (all published literature studied these hypotheses in patients with MCA stroke) is still under discussion. Whereas Lee et al $^{1}$ observed better outcomes in patients with distal FHV, several studies observed worse functional outcomes in patients with positive FHV signs. ${ }^{11,15,17,33}$ These in part conflicting findings may result from different methodologic approaches. For example, Lee et al or Haussen et $\mathrm{al}^{21}$ evaluated the significance of hyperintense vessels distal to the clot in occlusion of the MCA; Schellinger et $\mathrm{al}^{8}$ published a study on several MR imaging vessel signs; and other investigators focused the prognostic value of the FHV sign, depending more on its presence or absence than on quantitative criteria. ${ }^{11,17}$

The baseline DWI pc-ASPECTS as a formerly described reliable and easy-to-apply scoring system for the prediction of patient outcome and survival ${ }^{26,36}$ is confirmed by our data. Significantly higher pc-ASPECTSs were observed in patients with a good functional outcome and in patients who survived. Other DWI lesion scoring systems for BAO were not considered in our study, either because they do not account for supratentorial ischemic lesions ${ }^{37}$ or because their increased complexity was considered to be a potential source of error in the image analysis. ${ }^{38,39}$

Also, the occlusion site was confirmed to be a predictor of patient outcome. Whereas all patients with an occlusion of the proximal basilar artery died, survival rates of $82 \%$ with an occlusion in the midbasilar section and $100 \%$ with a thrombus at the basilar tip were observed, which supports the results of previous studies, for example, by Cross et $\mathrm{al}^{27}$ or Levy et $\mathrm{al}^{40}$ in which occlusions of the proximal basilar artery were considered to be 
associated with patient death (eg, a survival rate of $71 \%$ in patients with distal versus $15 \%$ in patients with proximal and midbasilar clots reported by Cross et al). The postinterventional TICI score, time from symptom onset to imaging, and time from imaging to DSA were not related to patient outcome in our study in contrast to the reported data in earlier publications. ${ }^{27,40,41}$

Limitations of our study are the small patient number and its retrospective design. In addition, the lack of uniformity in the treatment regimens, ranging from intra-arterial rtPA with or without thrombus fragmentation to thrombus aspiration to stent retrievers, is a potential source of error. Furthermore, MR images were acquired on 3 different scanner types (including 1.5T and 3T). To date, no literature has been published concerning possible differences in the occurrence of the FHV sign at different field strengths. These issues should be evaluated in further studies on larger patient collectives and retrospective analyses of standardized stroke registries with a long-term recording of patient outcome.

\section{CONCLUSIONS}

The FHBA sign proved to be a reliable, moderately sensitive, but specific and robust sign of basilar artery occlusion that may have significant implications for the correct diagnosis of this potentially fatal condition. It is associated with more extensive DWI lesion patterns and may be helpful in the prediction of patient survival. However, larger patient studies are necessary to address these hypotheses.

Disclosures: Karl-Titus Hoffmann-UNRELATED: Expert Testimony: Prosecution of the City of Leipzig, Law Courts of the Cities of Dresden and Dessau, Payment for Lectures (including service on Speakers Bureaus): Speaker for Bracco Imaging and Bayer Healthcare.

\section{REFERENCES}

1. Lee KY, Latour LL, Luby M, et al. Distal hyperintense vessels on FLAIR. Neurology 2009;72:1134-39

2. Azizyan A, Sanossian N, Mogensen MA, et al. Fluid-attenuated inversion recovery vascular hyperintensities: an important imaging marker for cerebrovascular disease. AJNR Am J Neuroradiol 2011;32:1771-75

3. Chen SP, Fuh JL, Lirng JF, et al. Hyperintense vessels on FLAIR imaging in reversible cerebral vasoconstriction syndrome. Cephalalgia 2012;32:271-78

4. Iancu-Gontard D, Oppenheim C, Touzé E, et al. Evaluation of hyperintense vessels on FLAIR MRI for the diagnosis of multiple intracerebral arterial stenoses. Stroke 2003;34:1886-91

5. Liu W, Xu G, Yue X, et al. Hyperintense vessels on FLAIR: a useful non-invasive method for assessing intracerebral collaterals. Eur J Radiol 2011;80:786-91

6. Kawashima M, Noguchi T, Takase Y, et al. Unilateral hemispheric proliferation of ivy sign on fluid-attenuated inversion recovery images in Moyamoya disease correlates highly with ipsilateral hemispheric decrease of cerebrovascular reserve. AJNR Am J Neuroradiol 2009;30:1709-16

7. Sanossian N, Saver JL, Alger JR, et al. Angiography reveals that fluidattenuated inversion recovery vascular hyperintensities are due to slow flow, not thrombus. AJNR Am J Neuroradiol 2009;30:564-68

8. Schellinger PD, Chalela JA, Kang D-W, et al. Diagnostic and prognostic value of early MR imaging vessel signs in hyperacute stroke patients imaged $<3$ hours and treated with recombinant tissue plasminogen activator. AJNR Am J Neuroradiol 2005;26:618-24

9. Wolf RL. Intraarterial signal on fluid-attenuated inversion recov- ery images: a measure of hemodynamic stress? AJNR Am J Neuroradiol 2001;22:1015-17

10. Cosnard G, Duprez T, Grandin C, et al. Fast FLAIR sequence for detecting major vascular abnormalities during the hyperacute phase of stroke: a comparison with MR angiography. Neuroradiology 1999;41:342-46

11. Kamran S, Bates V, Bakshi R, et al. Significance of hyperintense vessels on FLAIR MRI in acute stroke. Neurology 2000;55:265-69

12. Maeda M, Yamamoto T, Daimon S, et al. Arterial hyperintensity on fast fluid-attenuated inversion recovery images: a subtle finding for hyperacute stroke undetected by diffusion-weighted MR imaging. AJNR Am J Neuroradiol 2001;22:632-36

13. Tsushima Y, Endo K. Significance of hyperintense vessels on FLAIR MRI in acute stroke. Neurology 2001;56:1248-49

14. Toyoda K, Ida M, Fukuda K. Fluid-attenuated inversion recovery intraarterial signal: an early sign of hyperacute cerebral ischemia. AJNR Am J Neuroradiol 2001;22:1021-29

15. Girot M, Gauvrit JY, Cordonnier C, et al. Prognostic value of hyperintense vessel signals on fluid-attenuated inversion recovery sequences in acute cerebral ischemia. Eur Neurol 2007;57:75-79

16. Liu W, Yin Q, Yao L, et al. Decreased hyperintense vessels on FLAIR images after endovascular recanalization of symptomatic internal carotid artery occlusion. Eur J Radiol 2012;81:1595-600

17. Cheng B, Ebinger M, Kufner A, et al. Hyperintense vessels on acute stroke fluid-attenuated inversion recovery imaging associations with clinical and other MRI findings. Stroke 2012;43:2957-61

18. Pérez de la Ossa N, Hernández-Pérez $M$, Domènech $S$, et al. Hyperintensity of distal vessels on FLAIR is associated with slow progression of the infarction in acute ischemic stroke. Cerebrovasc Dis 2012;34:376-84

19. Huang X, Liu W, Zhu W, et al. Distal hyperintense vessels on FLAIR: a prognostic indicator of acute ischemic stroke. Eur Neurol 2012;68:214-20

20. Yoshioka K, Ishibashi S, Shiraishi A, et al. Distal hyperintense vessels on FLAIR images predict large-artery stenosis in patients with transient ischemic attack. Neuroradiology 2013;55:165-69

21. Haussen DC, Koch S, Saraf-Lavi E, et al. FLAIR distal hyperintense vessels as a marker of perfusion-diffusion mismatch in acute stroke. J Neuroimaging 2013;23:397-400

22. Olindo S, Chausson N, Joux J, et al. Fluid-attenuated inversion recovery vascular hyperintensity: an early predictor of clinical outcome in proximal middle cerebral artery occlusion. Arch Neurol 2012;69:1462-68

23. Connell L, Koerte IK, Laubender RP, et al. Hyperdense basilar artery sign: a reliable sign of basilar artery occlusion. Neuroradiology 2012;54:321-27

24. Rosset A, Spadola L, Ratib O. OsiriX: an open-source software for navigating in multidimensional DICOM images. J Digit Imaging 2004;17:205-16

25. Puetz V, Sylaja PN, Coutts SB, et al. Extent of hypoattenuation on CT angiography source images predicts functional outcome in patients with basilar artery occlusion. Stroke 2008;39:2485-90

26. Tei H, Uchiyama S, Usui T, et al. Posterior circulation ASPECTS on diffusion-weighted MRI can be a powerful marker for predicting functional outcome. J Neurol 2010;257:767-73

27. Cross DT, Moran CJ, Akins PT, et al. Relationship between clot location and outcome after basilar artery thrombolysis. AJNR Am J Neuroradiol 1997;18:1221-28

28. Higashida RT, Furlan AJ. Trial design and reporting standards for intra-arterial cerebral thrombolysis for acute ischemic stroke. Stroke 2003;34:e109-37

29. Gauvrit, Leclerc X, Girot M, et al. Fluid-attenuated inversion recovery (FLAIR) sequences for the assessment of acute stroke. J Neurol 2006;253:631-35

30. Mortimer AM, Saunders T, Cook J-L. Cross-sectional imaging for diagnosis and clinical outcome prediction of acute basilar artery thrombosis. Clin Radiol 2011:66:551-58 
31. Röther J, Wentz KU, Rautenberg W, et al. Magnetic resonance angiography in vertebrobasilar ischemia. Stroke 1993;24:1310-15

32. Vergouwen MD, Algra A, Pfefferkorn T, et al. Time is brain(stem) in basilar artery occlusion. Stroke 2012;43:3003-06

33. Ebinger M, Kufner A, Galinovic I, et al. Fluid-attenuated inversion recovery images and stroke outcome after thrombolysis. Stroke 2012;43:539-42

34. Sanossian N, Hao Q, Liebeskind DS. The thrombus and discontinuity of FLAIR vascular hyperintensity. Arch Neurol 2011;68:950-51

35. Hu XY, Ge ZF, Zee CS, et al. Differentiation of white and red thrombus with magnetic resonance imaging: a phantom study. Chin Med $J$ (Engl). 2012;125:1889-92

36. Nagel S, Herweh C, Köhrmann M, et al. MRI in patients with acute basilar artery occlusion: DWI lesion scoring is an independent predictor of outcome. Int J Stroke 2012;7:282-88

37. Cho TH, Nighoghossian N, Tahon F, et al. Brain stem diffusion- weighted imaging lesion score: a potential marker of outcome in acute basilar artery occlusion. AJNR Am J Neuroradiol 2009; 30:194-98

38. Renard D, Landragin N, Robinson A, et al. MRI-based score for acute basilar artery thrombosis. Cerebrovasc Dis 2008;25:511-16

39. Karameshev A, Arnold M, Schroth G, et al. Diffusion-weighted MRI helps predict outcome in basilar artery occlusion patients treated with intra-arterial thrombolysis. Cerebrovasc Dis 2011; 32:393-400

40. Levy EI, Firlik AD, Wisniewski S, et al. Factors affecting survival rates for acute vertebrobasilar artery occlusions treated with intraarterial thrombolytic therapy: a meta-analytical approach. Neurosurgery 1999;45:539-45

41. Brandt T, Kummer R von, Müller-Küppers M, et al. Thrombolytic therapy of acute basilar artery occlusion variables affecting recanalization and outcome. Stroke 1996;27:875-81 